\title{
Determination of an Aspergillus fumigatus-Specific Immunoglobulin G Reference Range in an Adult Omani Population
}

Munira Al-Rahman, ${ }^{1}$ Mahmood Al Kindi, ${ }^{1}$ Ibrahim Kutty, ${ }^{1}$ Ibrahim Al-Kalbani, ${ }^{2}$ *Jalila Alshekaili ${ }^{1}$

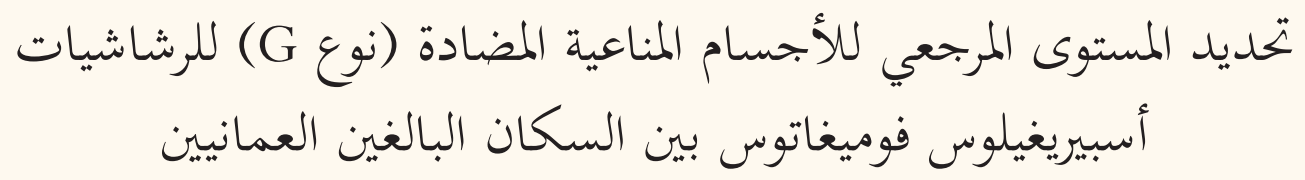

منيرة الرحمن، محمود الكندي، إبراهيم كوتي، إبراهيم الكلباني، جليلة الشكيلي

ABSTRACT: Objectives: The presence of abnormally high levels of Aspergillus fumigatus-specific immunoglobulin (Ig) $\mathrm{G}$ antibodies can serve as a diagnostic criterion for severe conditions like allergic bronchopulmonary and chronic pulmonary aspergillosis. This study aimed to determine a reference range of $A$. fumigatus-specific IgG levels within a healthy adult Omani population. Methods: This study took place during November 2015 at the Sultan Qaboos University Hospital, Muscat, Oman. The sera of 125 healthy Omani blood donors were tested for A. fumigatusspecific IgG levels using an automated fluorescence enzyme immunoassay. Results: Initially, the data were not normally distributed; however, log transformation and the exclusion of four outliers resulted in an acceptable Gaussian distribution. The reference range was $2.0-68.7 \mathrm{mg}_{\mathrm{A}} / \mathrm{L}$ at the $2.5^{\text {th }}$ and $97.5^{\text {th }}$ percentiles, respectively, with $90 \%$ confidence intervals of $2.0-3.0 \mathrm{mg}_{\mathrm{A}} / \mathrm{L}$ and $48.0-76.0 \mathrm{mg}_{\mathrm{A}} / \mathrm{L}$, respectively. Conclusion: The A. fumigatusspecific IgG reference range within a healthy adult Omani population was comparable to those reported in other populations.

Keywords: Aspergillus fumigatus; Allergic Bronchopulmonary Aspergillosis; Pulmonary Aspergillosis; Immunoglobulin G; Reference Range; Oman.

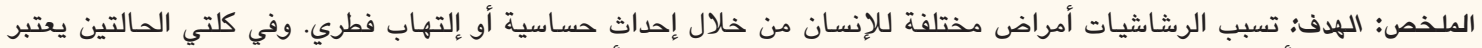

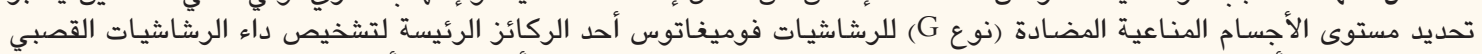

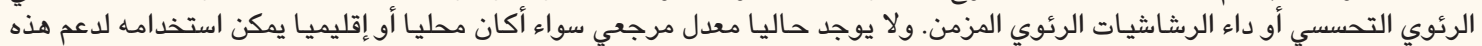

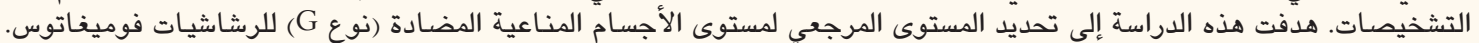

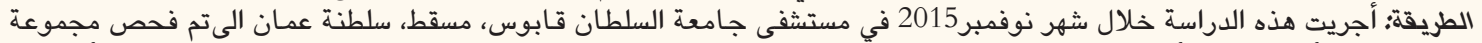

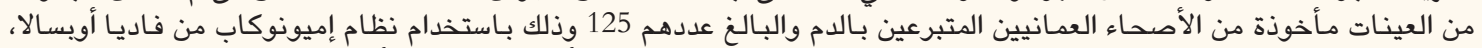

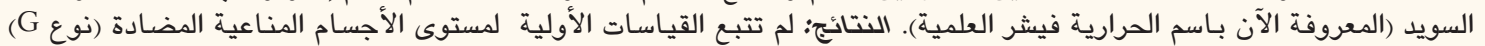

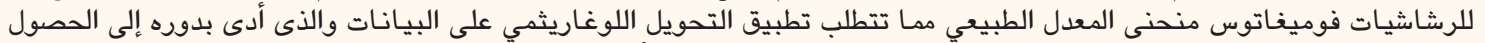

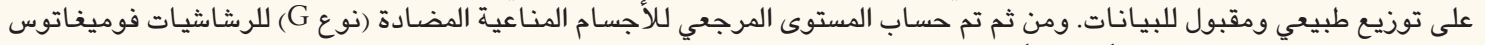

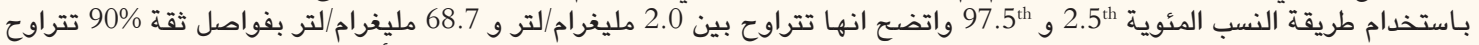

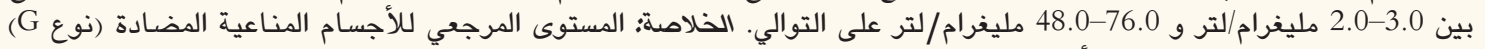

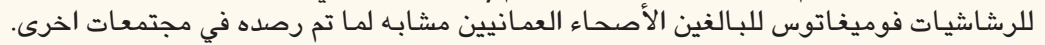

الكلمات المفتاحية: الرشاشيات فوميفاتوس؛ داء الرشاشيات القصبي الرئوي التحسسي؛ داء الرشاشيات الرئوي المزمن؛ المستوى المرجعي؛ عمان.

\section{Advances In KNOWLedge}

To the best of the authors' knowledge, this study is the first in Oman and the Arabian Gulf region to evaluate the reference range of Aspergillus fumigatus-specific immunoglobulin G (IgG) levels in a local population.

\section{Application to Patient Care}

The findings of the current study may guide clinicians in establishing diagnoses of allergic bronchopulmonary aspergillosis and chronic pulmonary aspergillosis at the Sultan Qaboos University Hospital, Muscat, Oman.

\section{$\mathrm{T}$} He Aspergillus genus is an extremely abundant type of airborne fungi constantly inhaled by humans which, for the most part, does not cause significant illness; however, individuals with pre-existing lung pathologies or weak- ened immune systems can develop Aspergillus-related diseases known as aspergillosis. ${ }^{1}$ Most cases of aspergillosis are caused by $A$. fumigatus (80\%), although other species can also cause infection, such as A. flavus, A. terreus and $A$. niger. ${ }^{1-3}$ Aspergillosis caused by 
A. fumigatus presents with either allergies-including allergic Aspergillus sinusitis and allergic bronchopulmonary aspergillosis (ABPA)—or infections, such as local aspergillomas affecting the lungs and sinuses, necrotising chronic pulmonary aspergillosis (CPA) and systemic invasive aspergillosis. ${ }^{4-6}$

Usually, ABPA or CPA is diagnosed following assessment of the patient's medical history for risk factors, a physical examination, radiological imaging and laboratory investigations. To this end, elevated levels of A. fumigatus-specific immunoglobulin (Ig) G antibodies are considered the main clinical criterion to support the diagnosis. ${ }^{4}$ However, there is currently no published reference range of anti-A. fumigatus-specific IgG levels for healthy adult populations in Arab countries. Therefore, the aim of this study was to establish a reference range for A. fumigatus-specific IgG antibodies in an Omani population. Hopefully, this reference range will guide clinicians in Oman when establishing diagnoses of ABPA and CPA.

\section{Methods}

This study took place in November 2015 at the Sultan Qaboos University Hospital (SQUH) in Muscat, Oman. Samples of sera were collected from 125 healthy adult donors attending the SQUH Blood Bank during the study period. Only healthy adult donors without any previous history of lung disease or asthma and no prior significant exposure to birds were included. Four outliers were excluded from the initial sample; however, the final sample of 121 subjects complied with the minimum sample size recommended by the Clinical and Laboratory Standards Institute (CLSI) for establishing a reference range..$^{7-9}$

A total of 5-10 mL of blood was obtained from each subject. The sera were immediately separated and stored at $-80{ }^{\circ} \mathrm{C}$ until analysis. Specific IgG antibodies against $A$. fumigatus were determined using an automated fluorescence enzyme immunoassay (Immuno-
$\mathrm{CAP}^{\circledR}$ system, Thermo Fisher Scientific Inc., Phadia AB, Uppsala, Sweden), as per the manufacturer's instructions. The assay was performed by an experienced senior biomedical scientist in the Immunology Laboratory of SQUH. Results were expressed as milligrams of Aspergillus-specific IgG antibodies per litre $\left(\mathrm{mg}_{\mathrm{A}} / \mathrm{L}\right)$. All reagents were received from the same lot number and each run included internal quality control samples. The interassay coefficient of variation between runs for the quality control samples was $<10 \%$, as per the manufacturer's instructions.

Data were analysed using the MedCalc ${ }^{\circledR}$ statistical software package, Version 16.4.3 (MedCalc Software, Ostend, Belgium). A non-parametric percentile method following the CLSI guidelines was used on log-transformed data to establish the reference range at a $90 \%$ confidence interval (CI). ${ }^{8,9}$ The level of statistical significance was set at $P<0.050$.

Ethical approval for this study was obtained from the Research \& Ethics Committee of the College of Medicine \& Health Sciences, Sultan Qaboos University (MREC \#1220-A). All subjects gave informed written consent prior to their participation in the study.

\section{Results}

The median age of the subjects was 24 years (range: 18-52 years old). Most of the donors were male, with a female-to-male ratio of 1:9.3. Initially, a frequency histogram and quantile-quantile plot to assess the distribution of the collected data indicated that the data were not normally distributed [Figure 1]. This finding was further supported by the results of a D'AgostinoPearson test $(P<0.001)$. Accordingly, the data were log-transformed for subsequent analysis. However, the data were still not normally distributed [Figure 2]. A Tukey's test identified four outliers, the exclusion of which resulted in the normal Gaussian distribution of the log-transformed data of the remaining 121 samples [Figure 3]. The reference range of $A$. fumigatus-specific
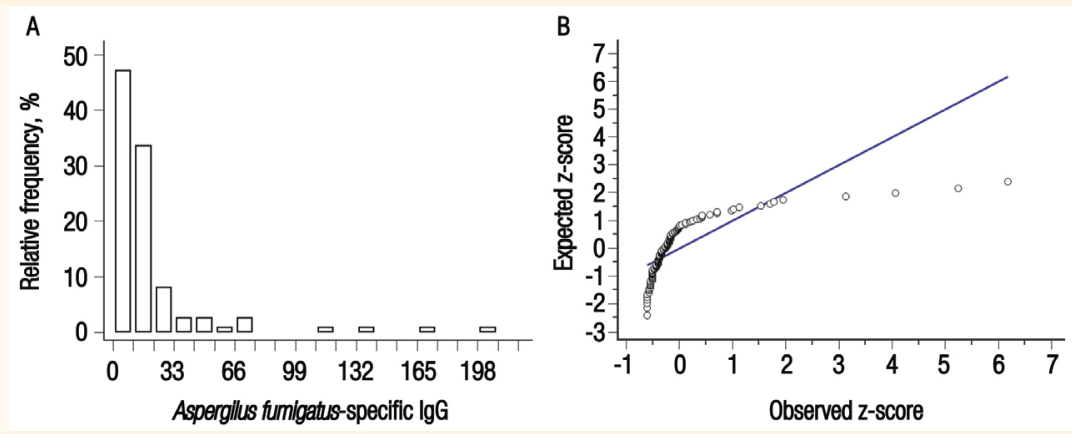

Figure 1: A: Relative frequency histogram of Aspergillus fumigatus-specific immunoglobulin G (IgG) antibodies in the sera of healthy Omani blood donors $(\mathrm{N}=125)$. B: Quantile-quantile plot of results obtained for A. fumigatus-specific IgG antibodies in the sera of healthy Omani blood donors $(\mathrm{N}=125)$.

IgG = immunoglobulin $G$. 

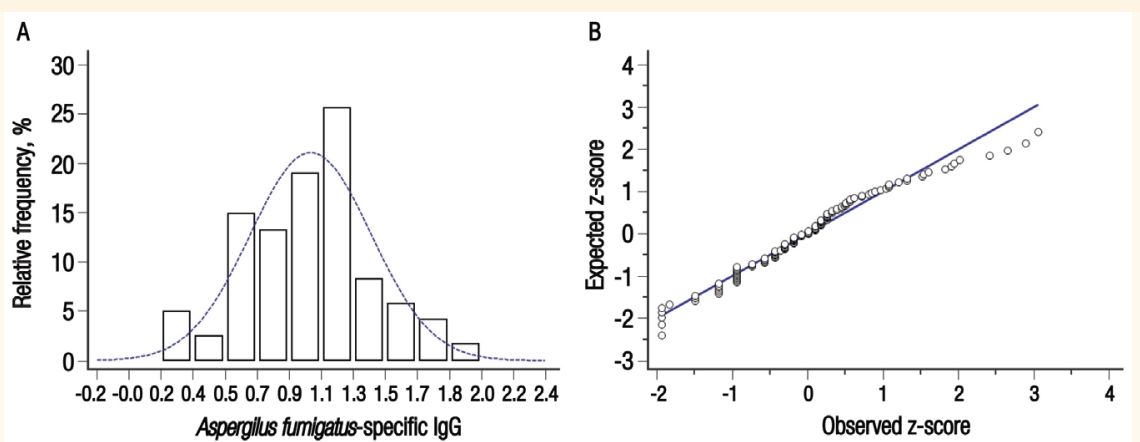

Figure 2: A: Relative frequency histogram of log-transformed Aspergillus fumigatus-specific immunoglobulin G (IgG) antibodies in the sera of healthy Omani blood donors $(\mathrm{N}=125)$. B: Quantile-quantile plot of log-transformed data for A. fumigatus-specific IgG antibodies in the sera of healthy Omani blood donors $(\mathrm{N}=125)$.

IgG = immunoglobulin $G$.
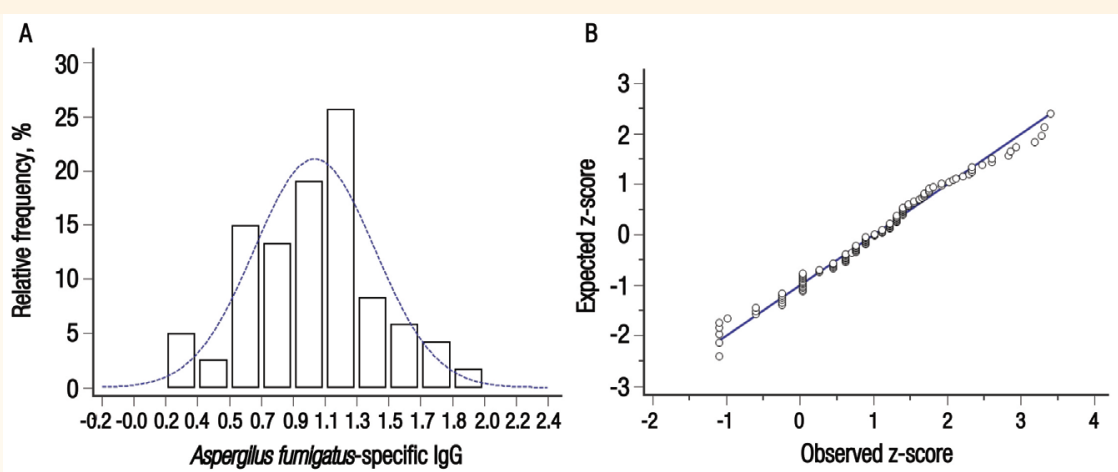

Figure 3: A: Relative frequency histogram of log-transformed Aspergillus fumigatus-specific immunoglobulin G (IgG) antibodies in the sera of healthy Omani blood donors $(\mathrm{N}=121)$. B: Quantile-quantile plot of log-transformed data for A. fumigatus-specific IgG antibodies in the sera of healthy Omani blood donors $(\mathrm{N}=121)$.

IgG = immunoglobulin $G$.

IgG for these 121 samples was $2.0-76.0 \mathrm{mg}_{\mathrm{A}} / \mathrm{L}$, with a geometric mean of $10.9 \mathrm{mg}_{\mathrm{A}} / \mathrm{L}$ and median of $11.0 \mathrm{mg}_{\mathrm{A}} / \mathrm{L}$. The $2.5^{\text {th }}$ percentile was $2.0 \mathrm{mg}_{\mathrm{A}} / \mathrm{L}$ with a $90 \% \mathrm{CI}$ of $2.0-3.0 \mathrm{mg}_{\mathrm{A}} / \mathrm{L}$ and the $97.5^{\text {th }}$ percentile was $68.7 \mathrm{mg}_{\mathrm{A}} / \mathrm{L}$ with a $90 \% \mathrm{CI}$ of $48.0-76.0 \mathrm{mg}_{\mathrm{A}} / \mathrm{L}$.

\section{Discussion}

Although uncommon, both ABPA and CPA are serious diseases that can affect immunocompetent patients with underlying lung pathologies. The former is a severe allergic and inflammatory condition that predominantly affects patients with asthma or cystic fibrosis and presents with fever, cough, shortness of breath, wheezing and haemoptysis; moreover, patients become sensitised to A. fumigatus, causing significant morbidity and mortality. ${ }^{10}$ Immunological mechanisms in ABPA that result in pathology are mainly type I immediate and type III immune complex hypersensitivity reactions mediated by IgE and IgG antibodies, respectively. ${ }^{4}$ The primary treatment for ABPA is oral corticosteroids to control the inflammatory process, while anti-fungals are used only as an adjuvant therapy in order to reduce the fungal load. ${ }^{9}$
In contrast, $\mathrm{CPA}$ is a chronic lung infection that can progress to pulmonary fibrosis and loss of lung function; the disease presents with constitutional and respiratory symptoms including weight loss, fatigue, shortness of breath and haemoptysis. ${ }^{10}$ In general, CPA affects immunocompetent patients with preexisting lung conditions as opposed to invasive aspergillosis, which usually occurs in immunocompromised patients. ${ }^{11}$ Underlying lung comorbidities which may predispose a patient to CPA include chronic obstructive pulmonary disease, pre-existing pulmonary cavitary tuberculosis, fibrotic sarcoidosis and ABPA. In some cases, CPA can manifest as a simple aspergilloma, chronic cavitary pulmonary aspergillosis, chronic fibrosing pulmonary aspergillosis, subacute invasive aspergillosis and aspergillosis nodules. ${ }^{11,12}$ For most patients, CPA is treated with antifungal agents and surgery. However, interferon- $\gamma$ has been recently proposed as an adjuvant therapy. ${ }^{4}$

In the current study, the reference range of A. fumigatus-specific IgG levels among a sample of healthy adult Omani blood donors was found to be 2.0-68.7 $\mathrm{mg}_{\mathrm{A}} / \mathrm{L}$ using the percentile method. This reference range was comparable to other published ranges 
and can potentially be used in conjunction with other parameters to diagnose patients suspected of having ABPA or CPA. Utilising the same immunoassay as that of the current study, Watkins et al. found the reference range of $A$. fumigatus-specific IgG levels to be 2.79-66.45 $\mathrm{mg}_{\mathrm{A}} / \mathrm{L}$ in a group of 122 healthy adults in South Africa. ${ }^{13}$ The same method and assay technology was used to detect an A. fumigatus-specific IgG range of $2.01-70.10 \mathrm{mg}_{\mathrm{A}} / \mathrm{L}$ in a group of 42 healthy individuals in Belgium. ${ }^{14}$ An enzyme-linked immunosorbent assay yielded similar results when assessing A. fumigatus IgG levels among 100 healthy Finnish children between $7-13$ years old to the $95^{\text {th }}$ percentile (67.8-68.3 $\mathrm{mg} / \mathrm{L})$; in addition, the researchers observed that $A$. fumigatus-specific IgG concentrations increased with age to reach a plateau at 6-7 years old, although the concentrations were not affected by gender. ${ }^{15}$ Agarwal et al. compared 67 patients with ABPA to 32 asthmatic controls in India; using a receiver operated characteristic curve, the researchers determined the ideal cut-off value for $A$. fumigatus-specific IgG to be 26.9 $\mathrm{mg}_{\mathrm{A}} / \mathrm{L} \cdot{ }^{16}$ However, it is important to note that the controls in this study were a small cohort of diseased rather than healthy individuals. ${ }^{16}$

To the best of the authors' knowledge, this study is the first in the Arabian Gulf region to derive a reference range for A. fumigatus-specific IgG levels in a cohort of healthy donors. In addition, the sample size was adequate in comparison to other studies and complied with CLSI recommendations. ${ }^{8,9,17}$ Nevertheless, this study was limited in that patients with ABPA or CPA were not included. Such patients should therefore be included in future research so as to validate the reference range determined. In addition, due to a lack of female patients within the cohort, no differences in A. fumigatus-specific IgG levels were noted between genders.

\section{Conclusion}

In summary, A. fumigatus-specific IgG levels are an important diagnostic parameter for patients with ABPA or CPA. In the current study, the reference range of A. fumigatus-specific IgG levels in healthy Omani donors was found to be $2.0-68.7 \mathrm{mg}_{\mathrm{A}} / \mathrm{L}$ at the $2.5^{\text {th }}$ and $97.5^{\text {th }}$ percentiles, respectively. This reference range was comparable to other published ranges reported from different populations worldwide.

\section{CONFLICT OF INTEREST}

The authors declare no conflicts of interest.

\section{References}

1. Barnes PD, Marr KA. Aspergillosis: Spectrum of disease, diagnosis, and treatment. Infect Dis Clin North Am 2006; 20:545-61. doi: 10.1016/j.idc.2006.06.001

2. Greenberger PA. Allergic bronchopulmonary aspergillosis. J Allergy Clin Immunol 2002; 110:685-92. doi: 10.1067/mai.20 02.130179 .

3. Nierman WC, Pain A, Anderson MJ, Wortman JR, Kim HS, Arroyo J, et al. Genomic sequence of the pathogenic and allergenic filamentous fungus Aspergillus fumigatus. Nature 2005; 438:1151-6. doi: 10.1038/nature04332.

4. Patterson KC, Strek ME. Diagnosis and treatment of pulmonary aspergillosis syndromes. Chest 2014; 146:1358-68. doi: 10.13 78/chest.14-0917.

5. Agarwal R, Sehgal IS, Dhooria S, Aggarwal AN. Developments in the diagnosis and treatment of allergic bronchopulmonary aspergillosis. Expert Rev Respir Med 2016; 10:1317-34. doi: 10.10 80/17476348.2016.1249853.

6. Denning DW, Cadranel J, Beigelman-Aubry C, Ader F, Chakrabarti A, Blot S, et al. Chronic pulmonary aspergillosis: Rationale and clinical guidelines for diagnosis and management. Eur Respir J 2016; 47:45-68. doi: 10.1183/13993003.005 83-2015.

7. Hyltoft Petersen P, Rustad P. Prerequisites for establishing common reference intervals. Scand J Clin Lab Invest 2004; 64:285-92. doi: 10.1080/00365510410006298.

8. Horn PS, Pesce AJ. Reference intervals: An update. Clin Chim Acta 2003; 334:5-23. doi: 10.1016/S0009-8981(03)00133-5.

9. Clinical and Laboratory Standards Institute. EP28: Defining, establishing, and verifying reference intervals in the clinical laboratory, 3rd ed. From: https://clsi.org/standards/products/ method-evaluation/documents/ep28/ Accessed: Nov 2017

10. Shah A, Panjabi C. Allergic bronchopulmonary aspergillosis: A perplexing clinical entity. Allergy Asthma Immunol Res 2016; 8:282-97. doi: 10.4168/aair.2016.8.4.282

11. Patterson TF, Thompson GR 3rd, Denning DW, Fishman JA, Hadley S, Herbrecht R, et al. Practice guidelines for the diagnosis and management of aspergillosis: 2016 update by the Infectious Diseases Society of America. Clin Infect Dis 2016; 63:e1-60. doi: 10.1093/cid/ciw326.

12. Hayes GE, Novak-Frazer L. Chronic pulmonary aspergillosis: Where are we? And where are we going? J Fungi (Basel) 2016; 2:18. doi: $10.3390 /$ jof 2020018

13. Watkins ML, Benjamin RL, Kotzé E, Hawarden D. Reference range for specific IgG antibodies to Aspergillus fumigatus in the South African adult population. Curr Allergy Clin Immunol 2012; 25:212-14.

14. Van Hoeyveld E, Dupont L, Bossuyt X. Quantification of IgG antibodies to Aspergillus fumigatus and pigeon antigens by ImmunoCAP technology: An alternative to the precipitation technique? Clin Chem 2006; 52:1785-93. doi: 10.1373/clinchem.2006.067546.

15. Korppi M, Laitinen S, Taskinen T, Reiman M, Nevalainen A, Husman T. Mold-specific immunoglobulin G antibodies in a child population. Pediatr Allergy Immunol 2003; 14:371-7. doi: 10.10 34/j.1399-3038.2003.00059.x.

16. Agarwal R, Dua D, Choudhary H, Aggarwal AN, Sehgal IS, Dhooria S, et al. Role of Aspergillus fumigatus-specific IgG in diagnosis and monitoring treatment response in allergic bronchopulmonary aspergillosis. Mycoses 2017; 60:33-9. doi: 10.11 11/myc.12541.

17. Hyltoft Petersen P, Rustad P. Prerequisites for establishing common reference intervals. Scand J Clin Lab Invest 2004; 64:285-92. doi: 10.1080/00365510410006298.

\section{FUNDING}

No funding was received for this study. 